\title{
面向节能的工艺规划与调度集成问题建模研究*
}

\author{
孟否磊 张超勇 邵新宇 任亚平 \\ (华中科技大学数字制造装备与技术国家重点实验室＼cjkstart武汉４30074)
}

\begin{abstract}
摘要: 工艺规划和车间调度是离散制造车间中的两大关键环节, 将两者集成优化有助于提高车间生产效率、降低能源消耗。 研究以节能为目标的工艺规划与调度集成(Integration of process planning and scheduling, IPPS)问题, 基于空闲时间与空闲能耗 两种建模思想, 建立 3 个考虑关机重启节能策略的混合整数线性规划(Mixed integer linear programming, MILP)模型。从模型 尺寸复杂度、计算复杂度等方面对这三个 MILP 模型进行详细的对比评估。通过使用 CPLEX 求解器对 IPPS 调度实例进行求 解, 证明了所提出 MILP 模型的正确性与有效性。试验结果表明基于不同建模思路的 MILP 模型尺寸复杂度、计算复杂度差 别很大, 基于空闲能耗的 MILP 模型求解效果好于基于空闲时间的 MILP 模型。
\end{abstract}

关键词: 工艺规划与调度集成; 混合整数线性规划; 节能; 关机重启策略

中图分类号: TP18

\section{Mathematical Modeling of Energy-efficient Integration of Process Planning and Scheduling}

\author{
MENG Leilei ZHANG Chaoyong SHAO Xinyu REN Yaping \\ (State Key Lab of Digital Manufacturing Equipment and Technology, \\ Huazhong University of Science and Technology, Wuhan 430074)
}

\begin{abstract}
Process planning and scheduling are two main parts of the discrete manufacturing workshops, and integrating these two parts will help to increase production efficiency and reduce energy consumption. The integration of process planning and scheduling (IPPS) problem with the objective of minimizing energy consumption is studied. Three mixed integer linear programming (MILP) models that consider turning Off \On strategy are proposed based on two different modeling ideas namely idle time and idle energy. These three models are compared and evaluated detailedly under both the size and computational complexities. The correctness and effectiveness of the proposed models are verified by using CPLEX solver to solve the instances of IPPS. The experimental results show that the MILP models based on different modeling ideas are very different in both the size and computational complexities. The model based on the modeling idea of idle energy is better than the models based on the modeling idea of idle time.
\end{abstract}

Key words: integration of process planning and scheduling; mixed integer linear programming; energy-saving; turning Off $\backslash$ On strategy

\section{0 前言}

随着社会、经济、科技的发展，人类对石油、 煤炭等化石燃料的需求也日益扩大, 但地球上的这 些资源是有限的, 已日益趋于枯竭, 因此节能变得 越来越重要。制造企业是高能耗企业, 制造业消耗 的能源将近占全球总能源的 1/3, 产生了全球 36\% 的 $\mathrm{CO}_{2}{ }^{[1]}$ 。数控机床是制造企业的母机, 能源消耗

* 国家自然科学基金(51575211)、中美基金委国际(地区)合作与交流 (51861165202)和吉林省自然科学基金(20180101058JC) 资助项目。 20181102 收到初稿, 20190524 收到修改稿
巨大, 据统计, 我国拥有机床大约 800 多万台, 居 世界第一，其耗电量是非常惊人的 ${ }^{[2]}$ 。然而，机床 在实际加工过程中, 大部分时间是处于空载待机状 态, 而非用于加工, 据统计机床大约 $80 \%$ 的能耗消 耗在待机以及关机重启过程中, 真正用于加工的能 耗所占比例不足 $20 \%{ }^{[3]}$, GUTOWSKI 等 ${ }^{[4]}$ 给出的一 条汽车自动生产线的能量效率竟然只有 $14.8 \%$ 。可 见，制造车间节能潜力巨大。

目前, 针对车间节能方面的研究主要从机床节 能设计与管理两方面入手。机床节能设计, 就是通 过各种节能技术来降低机床自身能耗部件的损耗, 如通过轻量化设计等降低机床各部分零件质量, 减 
少能耗部件自身的动能损耗 ${ }^{[5]}$ 。通过机床电机额定 功率与实际功率的合理匹配, 减少电机额外损耗; 通过使用高能效的变频器等, 减少机床的电器损耗 等。在管理方面, 主要通过切削参数优化 ${ }^{[6,7]}$ 、工艺 路线规划 ${ }^{[8]}$ 、车间调度 ${ }^{[9]}$ 以及它们之间的集成来降 低能耗 ${ }^{[9-11]}$ 。

工艺规划和车间调度是离散制造车间中两大关 键环节，两者集成优化能更好提高车间生产效率、 降低能源消耗。工艺规划与调度集成(Integration of process planning and scheduling, IPPS) 问题需要同时 考虑工艺路线选择、机器选择、工序排序三个子问 题, 是一个比单独的工艺规划问题与调度问题更为 复杂的 NP-Hard 问题。求解该问题的算法主要包括 精确算法、启发式算法以及元启发式算法。数学规 划方法, 如混合整数线性规划(Mixed integer linear programming, MILP)模型是求解该问题一个常用的 精确方法。文献[12-14]分别建立了求解该问题的 MILP 模型, 都是假设每个工件的所有工艺路线都 已生成，但是针对工艺路线比较多的问题时，该方 法计算量太大。JIN 等 ${ }^{[13]}$ 针对这一缺陷, 基于 “组 合”与 “缩减组合” 两种概念以及 WAGNER 和 MANNE $^{[15]}$ 两种建模方法, 提出了 4 个基于网络图 的 IPPS 问题的 MILP 模型, 并对这 4 个模型进行 了详细的对比分析, 结果表明基于组合概念及 Manne 优先关系的建模方法的模型好于其他模型。 但是, MILP 模型局限于求解大规模问题时效率极

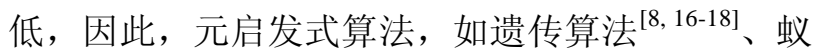
群算法 ${ }^{[19]}$ 、蜂群算法 ${ }^{[20]}$ 等被广泛用来解决 IPPS 问 题。虽然元启发式算法可以在较短时间内得到问题 较好的解, 但是需要针对具体问题设计不同的编 码、解码、邻域结构、更新策略等, 当算法设计的 不好时, 结果有可能也不理想。即使针对小规模 问题, 元启发式算法也不能保证得到问题的最优 解 (无法证明解的最优性), 而 MILP 模型等精确方 法对于小规模问题则可以得到最优解。

MILP 等数学模型的构建是认识优化问题的基 础, 它可以清楚的描述目标问题的目标、约束条件 等所有特征, 是挖掘相关领域知识与启发式规则的 关键所在 ${ }^{[21]}$ 。数学模型可以用分支定界、割平面、 分支剪枝等方法求解 ${ }^{[22]}$, 这些方法的求解效率与效 果依赖具体的模型, 但不同的模型复杂度不同, 如 决策变量数量、决策变量维数、约束方程个数以及 约束方程松紧程度不同。高效的模型具有更快的求 解效率, 在相同的时间内可以得到更好的结果, 因 此高效数学模型的构建非常有意义。随着计算机以 及数学模型求解软件性能的快速提升, 关于调度问
题建模方面的研究引起越来越多的关注。

至目前, 国内外针对 IPPS 问题的研究主要针对

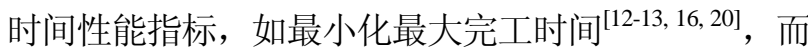
对车间能耗方面研究较少, 但随着能源的紧缺, 能源 价格的提高，以节能为目标制造车间调度问题逐步成 为热点。本文研究工艺规划与调度集成优化来减少车 间能耗, 就作者目前所知, 还没有以能耗最小化为目 标，同时考虑关机重启策略的 IPPS 问题的 MILP 模 型。本文根据 IPPS 问题自身的特点, 以能耗最小为 目标，同时考虑关机重启策略，依据空闲时间与空闲 能耗两种建模思想，建立 3 个 MILP 模型，并从建模 过程、尺寸复杂度与以及计算复杂度等方面对所提出 模型进行对比分析，验证提出模型的有效性。

\section{1 问题描述}

随着能源的紧缺, 能源价格的提高, 以节能为 目标的车间调度问题逐步成为热点。DAI 等 ${ }^{[8]}$ 同时 考虑最大完工时间与车间能耗两个目标, 提出了解 决该问题的多目标遗传算法并通过具体实例证明了 该方法的有效性。ZHANG 等 ${ }^{[19]}$ 研究了 IPPS 节能优 化问题, 提出了改进的蚁群算法来解决该问题, 实 验结果表明优化后的调度方案可以节约 $10.7 \%$ 的能 耗。ZHANG 等 ${ }^{[17]}$ 提出了以节能为目标的 IPPS 非 线性数学模型以及解决该问题的遗传算法。WANG 等 ${ }^{[23]}$ 研究了以能耗最小化为目标的切削参数、工艺 规划以及调度集成问题，并提出一系列节能策略。 $\mathrm{HE}$ 等 ${ }^{[24]}$ 提出了面向对象的 IPPS 能耗模型, 采用事 件图建模方法对加工过程能量流进行建模。何彦等 ${ }^{[25]}$ 以能耗、完工时间、机床负载为目标, 研究了 IPPS 多目标调度问题并提出了基于改进 $\mathrm{Q}$ 学习算法的求 解方案。刘琼等 ${ }^{[26]}$ 以碳排放和最大完工时间为目 标, 研究了基于 NSGA-II 多目标求解方法的 IPPS 问题。李聪波等 ${ }^{[27]}$ 研究了面向能耗的 IPPS 分批优 化调度问题，并采用多目标模拟退火算法来同时优 化碳排放和最大完工时间。

当机床连续待机时间比较长时, 对于允许使用 过程中关机的机床可以通过关机重启策略来节约 机床能耗。关机重启策略最早由 MOUZON 等 ${ }^{[3]}$ 提 出, 并成功应用到单机调度中。随后关机重启策略 被逐步应用到单机 ${ }^{[28-30]}$ 、并行机 ${ }^{[31]}$ 、作业车间 ${ }^{[32]}$ 、 柔性作业车间 ${ }^{[33]}$ 中。WANG 等 ${ }^{[34]}$ 将机床划分为待 机、加工等多个能耗状态, 基于 ARENA 仿真软件, 建立了离散加工系统层的多粒度能耗仿真模型, 仿 真结果表明, 实行关机重启策略可以节约 26\%的能 耗。MOUZON 等 ${ }^{[3]}$ 通过对非瓶颈机器实行关机重 
启策略, 避免机床长时间处于空闲状态, 结果表明 可以节约 $80 \%$ 的空闲能耗。CHE 等 ${ }^{[28]}$ 基于空闲能耗 的建模思想, 提出了考虑关机重启策略的单机调度 能耗 MILP 模型。LIANG 等 ${ }^{[31]}$ 以拖期与能耗为目标, 研究了考虑关机重启策略的不相关并行机调度问 题。ZHANG 等 ${ }^{[33]}$ 基于空闲时间的建模思想, 提出 了考虑关机重启策略的柔性作业车间调度问题的
MILP 模型, 并通过使用 CPLEX 求解器对小规模实 例进行求解，验证了其可行性。至目前，还没有以 能耗最小化为目标, 同时考虑关机重启策略的 IPPS 问题的 MILP 模型。因此, 本文旨至于建立求解该 问题的 MILP 模型，并验证它们的有效性。

\section{1 参数定义}

本文相关参数及其含义见表 1 。

表 1 相关参数及其含义

\begin{tabular}{|c|c|c|c|}
\hline 符号 & 参数含义 & 符号 & 参数含义 \\
\hline$i$ & 工件序号 & $K_{i, h, j}$ & 工序 $O_{i, h, j}$ 的可选机器集合 $\left\{1,2, \cdots, m_{i, h, j}\right\}$ \\
\hline$n$ & 工件总数 & $t$ & 机器位置序号 \\
\hline$I$ & 工件集合 $\{1,2, \cdots, n\}$ & $p_{k}$ & 机器 $k$ 最大可能的位置数 \\
\hline$j, j j$ & 工序序号 & $L_{k}$ & 机器 $k$ 的位置集合 $\left\{1,2, \cdots, p_{k}\right\}$ \\
\hline$S_{i}$ & 工件 $i$ 的工序数 & $p_{i, h, j, k}^{t}$ & 工序 $O_{i, h, j}$ 在机器 $k$ 上的加工时间 \\
\hline$J_{i}$ & 工件 $i$ 工序集合 $\left\{1,2, \cdots, S_{i}\right\}$ & $P_{i, h, j, k}$ & 工序 $O_{i, h, j}$ 在机器 $k$ 上的加工功率 \\
\hline$h$ & 组合序号 & $M$ & 一个极大正数 \\
\hline$h_{i}$ & 工件 $i$ 的组合数 & $P_{i d l e}^{k}$ & 机床 $k$ 的待机功率 \\
\hline$H_{i}$ & 工件 $i$ 的组合集合 $\left\{1,2, \cdots, h_{i}^{n}\right\}$ & $N_{k}$ & 机床 $k$ 在每次加工任务中所允许中途关机重启的次数 \\
\hline$S_{i, h}$ & 工件 $i$ 第 $h$ 个组合的工序数 & $E_{t u m}^{k}$ & 机床 $k$ 关机重启一次所消耗的能量 \\
\hline$J_{i, h}$ & 工件 $i$ 第 $h$ 个组合的工序集合 $\left\{1,2, \cdots, S_{i, h}\right\}$ & $T_{k}$ & 机床 $k$ 关机重启一次所需要的时间 \\
\hline$O_{i, h, j}$ & 工件 $i$ 第 $h$ 个组合中第 $j$ 道工序 & $T_{k}^{B}$ & 机床 $k$ 的空载平衡时间, 即实行关机重启策略所需要的最短时间 \\
\hline$k$ & 机床编号 & $y_{i, h, j, k}$ & $\begin{array}{l}0-1 \text { 常量, 当为 } 1 \text { 时, 表示工序 } O_{i, h, j} \text { 可以在机床 } k \text { 上加工, 否则, 工序 } O_{i, h, j} \\
\text { 不能在机床 } k \text { 上加工 }\end{array}$ \\
\hline$m$ & 机床总数 & $Q_{i, h, j, j}$ & $\begin{array}{l}\text { 优先约束常量, 当为 } 1 \text { 时, 表示在工件 } i \text { 的第 } h \text { 组合中, 工序 } O_{i, h, j} \text { 必须 } \\
\text { 在 } O_{i, h, j j} \text { 之前加工, 当为 } 2 \text { 时, 表示工序 } O_{i, h, j} \text { 与 } O_{i, h, j j} \text { 没有优先关系 }\end{array}$ \\
\hline$k$ & 所有机器集合 $\{1,2, \cdots, m\}$ & $P_{0}$ & 公共功率 \\
\hline$m_{i, h, j}$ & 工序 $O_{i, h, j}$ 的可选机器总数 & $C_{\max }$ & 最大完工时间 \\
\hline
\end{tabular}

\subsection{IPPS 问题描述}

IPPS 可描述为车间中有 $n$ 个待加工工件 $\{1,2, \cdots, n\}$, 其中工件 $i$ 有 $S_{i}$ 道加工工序, 这些工序 可选择在 $m$ 台机器 $\{1,2, \cdots, m\}$ 上进行加工, 存在同 一工件的某些工序之间没有先后约束关系或者某些 工件只需要部分工序加工的情况, 工件的某些工序 可选择在不同机床上加工。优化目标是给每个工件 选择最合适的加工工序以及确定这些工序之间的先 后关系, 给每道工序分配最合适的加工机器并且确 定各机器上所有加工工序的最优加工次序、开始时 间，使得车间总能耗目标最小。

该问题需满足以下基本假设: 所有机器、工件 在 0 时刻都可用; 任一工件在同一时间只能在一台 机器上加工, 任一机器在同一时刻最多只能加工一 个工件; 如果某一工序已经开始加工, 则只能等到 该工序加工完成方可停止; 不同工件的工序之间是 独立的, 没有先后关系约束; 忽略同一机器上不同
工件工件间的转换时间、以及同一工件不同阶段间 的运输时间。

\section{3 车间能耗}

车间总能耗大致可以划分为机床能耗、公共能 耗两部分, 机床能耗包括加工能耗以及待机能耗两 部分, 下面对各个能耗组成部分进行分析。

\subsection{1 加工能耗}

加工能耗指的是机床加工工件时所消耗的能 量 ${ }^{[35]}$, 总加工能耗 $T^{P C}$ 可表示为

$$
\begin{gathered}
T^{P C}=\sum_{i \in I} \sum_{h \in H_{i}} \sum_{j \in J_{i, h}} \sum_{k \in K_{i, h, j}} P_{i, h, j, k}^{E} D_{i, h, j, k}= \\
P_{i, h, j, k} p_{i, h, j, k}^{t} D_{i, h, j, k}
\end{gathered}
$$

式中, $P_{i, h, j, k}^{E}$ 表示工件 $i$ 第 $h$ 个组合中第 $j$ 道工序在 机床 $k$ 上的加工能耗; $D_{i, h, j, k}$ 为 0-1 变量, 表示工序 $O_{i, h, j}$ 是否选择在机床 $k$ 上加工, 当为 1 时, 表示选 择, 否则为 0 。 


\subsection{2 待机能耗}

待机能耗是指机床由于上一个工件已经加工 完, 下一个工件工件还未到达而出现闲置状态所消 耗的能量, 机床 $k$ 的待机能耗 $I_{k}^{E}$ 可表示为

$$
I_{k}^{E}=\sum_{t=1}^{p_{k}-1} I_{k, t}^{E}=\sum_{t=1}^{p_{k}-1} P_{i d l e}^{k}\left(S_{k, t+1}-F_{k, t}\right)
$$

式中, $I_{k, t}^{E}$ 表示机床 $k$ 第 $t$ 与 $t+1$ 位置的待机能耗; $F_{k, t}$ 表示机器 $k$ 上第 $t$ 个位置的结束时间; $S_{k, t}$ 表示 机器 $k$ 上第 $t$ 个位置的开始时间。

\subsection{3 机床总能耗}

机床总能耗 $T^{M C}$ 为总加工能耗以及所有机床待 机能耗之和, 其可表示为

$$
\begin{gathered}
T^{M C}=T^{P C}+\sum_{k \in K} I_{k}^{E}=P_{i, h, j, k} p_{i, h, j, k}^{t} D_{i, h, j, k}+ \\
\sum_{k \in K} \sum_{t=1}^{p_{k}-1} P_{i d l e}^{k}\left(S_{k, t+1}-F_{k, t}\right)
\end{gathered}
$$

当机床 $k$ 的某待机时间段 $S_{k, t+1}-F_{k, t}$ 比较长时, 可以实施关机重启策略, 节约机床能耗, 可以实行 关机重启策略的最短空闲时间, 即空载平衡时间 $T_{k}^{B}$ 为

$$
T_{k}^{B}=\max \left\{T_{k}, E_{\text {turn }}^{k} / P_{\text {idle }}^{k}\right\}
$$

式中, $E_{\text {tum }}^{k}$ 表示机床 $k$ 实行一次关机重启策略所需 要的能耗, 包括机床关机、开机、机床预热等阶段 所需要的能耗; $T_{k}$ 表示机床 $k$ 一次关机重启策略所 需要的时间, 包括一次关机、开机、预热等时间, 是从开始关机到机床恢复准备加工状态所需的所有 时间。空载平衡时间 $T_{k}^{B}$ 表示对机床 $k$ 实行一次关机 重启策略所需要的最短空闲时间，即当机床 $k$ 的一 个空闲时间段大于一次关机重启策略所需要的时 间 $T_{k}$, 且机器 $k$ 在该空闲时间段内所消耗的能量大 于机床 $k$ 一次关机重启所需要的能量 $E_{u u m}^{k}$ 时才可实 施关机重启策略。

引入关机重启策略后, 待机能耗 $I_{k, t}^{E}$ 可表示为

$$
I_{k, t}^{E}=\left(1-W_{k, t}\right)\left(S_{k, t+1}-F_{k, t}\right) P_{\text {idle }}^{k}+W_{k, t} E_{u u m}^{k}
$$

式中, $W_{k, t}$ 为 0-1 变量, 用来确定是否在机床的 $t$ 与 $t+1$ 位置间实行关机重启策略, 为 1 则实行, 否则 为 0 。

\subsection{4 公共能耗}

公共能耗 $C^{E}$, 是指机械加工车间中照明、通风、 空调等公共设备为维持车间环境消耗的能量, $C E$ 等于公共功率 $P_{0}$ 与最大完工时间 $C_{\text {max }}$ 的乘积,

$$
C^{E}=P_{0} C_{\max }
$$

\subsection{5 车间总能耗}

车间总能耗等于机床总能耗与公共能耗之和,
引入关机重启策略后, 车间总能耗 $T^{E C}$ 可表示为

$$
\begin{gathered}
T^{E C}=T^{M C}+C^{E}=P_{i, h, j, k} p_{i, h, j, k}^{t} D_{i, h, j, k}+ \\
\sum_{k \in K}^{p_{k}-1} \sum_{t=1}\left(\left(1-W_{k, t}\right)\left(S_{k, t+1}-F_{k, t}\right) P_{i d l e}^{k}+W_{k, t} E_{\text {turn }}^{k}\right)+P_{0} C_{\max }
\end{gathered}
$$

\section{MILP 模型构建}

本节建立 3 个 IPPS 节能问题的 MILP 模型， 3 个 MILP 模型都是基于机器位置的 WAGNER ${ }^{[36]}$ 建 模方法。基于机器位置的 WAGNER 建模方法, 建 立在“机床位置”概念上，即将一个机床按照时间先 后分成若干个段, 每一段即成为一个位置, 并且要 求每一个位置最多只能安排一个工序, 因此, 只要 确定了工序与机床位置的对应关系, 工件在机床上 的调度方案则可得到。对这 3 个 MILP 模型的建模 方法进一步划分可以分为两类，第一类是基于空闲 时间的建模方法, 包括模型 1 与模型 2 , 第二类是 基于空闲能耗的建模方法，包括模型 3。基于空闲 时间的建模方法是指机床待机能耗通过待机段时间 与待机功率来计算, 而基于空闲能耗的建模方法直 接定义空闲段能耗决策变量。

本文所有模型采用基于 “组合” 的概念 ${ }^{[13]}$ ，即 将含有 $\mathrm{OR}$ 节点的网络图拆分成多个不含 $\mathrm{OR}$ 节点 的网络图。如图 1 中, 工件 1 有 3 条可选工艺路线, 分别为 1-2-5,1-3-5 以及 1-4-5, 那么将工件 1 网络图 拆分为 3 个不带 $O R$ 节点的网络图, 即组合 1 , 组 合 2 以及组合 3 。工件 2 不同于工件 1 , 工件 2 的 2 、 3、4 道工序必须都加工, 但是之间没有先后关系。 工艺路线网络图中, OR 表示只能选其中一条; AND 表示所有的必须选择，但是它们之间没有先后约束 关系。

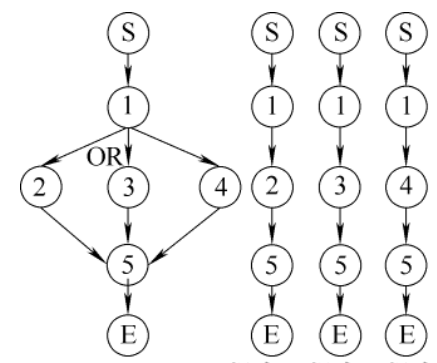

组合 1 组合 2 组合 3 工件 1

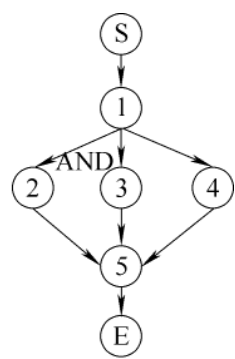

工件 2
图 1 工件 1 与工件 2 工艺路线网络图

一个完整的 MILP 模型包括决策变量、目标函 数、约束条件 3 部分。MILP 模型的求解效率由决 策变量以及约束条件决定, 按影响程度从大到小依 次为 $0-1$ 决策变量数、约束方程数及连续决策变量 
数 ${ }^{[37]}$ 。基于不同的建模方法, 模型的决策变量个数、 决策变量维数、约束方程数往往差别很大, 从而导 致模型求解效率也不一样。

\section{1 模型 1}

\subsection{1 决策变量}

模型 1 总共定义了 10 个决策变量, 其中, 决策 变量 $X_{i, h} 、 Y_{i, h, j, k, t} 、 Z_{i, j, j j} 、 W_{k, t}$ 为 $0-1$ 决策变量。 决策变量 $X_{i, h}$ 用于确定工件加工组合的选择; 决策 变量 $Y_{i, h, j, k, t}$ 用于确定工序与机床位置间的相应关 系。由于 $D_{i, h, j, k}$ 与 $Y_{i, h, j, k, t}$ 存在如式(8)所示关系, 因 此在模型中省去 $D_{i, h, j, k}$, 从而降低模型复杂度。

$$
D_{i, h, j, k}=\sum_{t \in L_{k}} Y_{i, h, j, k, t}, \forall i \in I, h \in H_{i}, j \in J_{i, h}, k \in K_{i, h, j}
$$

决策变量 $Z_{i, j, j j}$ 用于确定同一工件中无先后关 系的工序间的先后关系(如图 1 中工件 2 的第 2、3、 4 道工序); $W_{k, t}$ 用于确定在机床的某一个位置后是 否实行关机重启策略。连续决策变量 $B_{i, h, j}$ 与 $S_{k, t}$ 分 别用于表示工序的开始时间与机床位置的开始时 间。 $U_{k, t}$ 与 $V_{k, t}$ 为中间连续决策变量, 是为了将目标 函数线性化而引入的变量。 $A_{i, h, j, k, t}$ 为中间 0-1 决策 变量。 $C_{\text {max }}$ 为连续决策变量, 表示最大完工时间。

$$
\begin{gathered}
X_{i, h}=\left\{\begin{array}{l}
1, \text { 若工件 } i \text { 选择第 } h \text { 个加工组合 } \\
0, \text { 否则 }
\end{array}\right. \\
Y_{i, h, j, k, t}=\left\{\begin{array}{l}
1, \text { 若工序 } O_{i, h, j} \text { 安排在机器 } k \text { 的 } \\
\text { 第 } t \text { 个位置上加工 } \\
0, \text { 否则 }
\end{array}\right. \\
Z_{i, j, j j}=\left\{\begin{array}{l}
1, \text { 若工序 } O_{i, h, j} \text { 先与工序 } O_{i, h, j, j} \text { 加工 } \\
0, \text { 否则 }
\end{array}\right. \\
W_{k, t}=\left\{\begin{array}{l}
1, \text { 若机器 } k \text { 第 } t \text { 到 } t+1 \text { 位置间 } \\
0, \text { 否则 }
\end{array}\right.
\end{gathered}
$$

\subsection{2 目标函数}

$$
\begin{gathered}
\min T^{E C}=\sum_{k \in K} \sum_{t=1}^{p_{k}-1}\left(( 1 - W _ { k , t } ) \left(S_{k, t+1}-\right.\right. \\
\left.\left.\left(S_{k, t}+\sum_{i \in I} \sum_{h \in H_{i}} \sum_{j \in J_{i, h}}\left(p_{i, h, j, k}^{t} Y_{i, h, j, k, t}\right)\right)\right) P_{i d l e}^{k}+E_{u u m}^{k} W_{k, t}\right)+ \\
\sum_{i \in I} \sum_{h \in H_{i}} \sum_{j \in J_{i, h}} \sum_{k \in K_{i, h, j}} \sum_{t \in L_{k}} P_{i, h, j, k} p_{i, h, j, k}^{t} Y_{i, h, j, k, t}+P_{0} C_{\max }
\end{gathered}
$$

目标函数中第一项表示机床待机能耗加上关机 重启所需要的能耗, 第二项为加工能耗, 第三项为 公共能耗。由目标函数可以看出, 目标函数是非线 性的, 存在决策变量相乘的情况 $\left(1-W_{k, t}\right) S_{k,+1}$ 、
$\left(1-W_{k, t}\right) S_{k, t}$ 以及 $\left(1-W_{k, t}\right) Y_{i, h, j, k, t}$, 因为非线性模型求 解非常复杂, 因此, 需要对模型进行转换, 将非线 性目标函数转换为线性的。本文通过引入中间决策 变量 $U_{k, t} 、 W_{k, t}$ 以及 $A_{i, h, j,}$, 用 $U_{k, t+1}$ 代替 $\left(1-W_{k, t} S\right)_{k t}$, 用 $V_{k, t}$ 代替 $\left(1-W_{k, t}\right) S_{k, t}$, 用 $A_{i, h, j, t}$ 代 替 $\left(1-W_{k, t}\right) Y_{i, h, j, k, t}$, 然后通过添加约束 $(26) \sim(36)$, 保证 $U_{k, t}=\left(+Z_{k, t}\right) S_{+k} 、 V_{k, t}=\left(1-Z_{k, t}\right) F_{k, t}$ 以及 $A_{i, h, j, \bar{k}, t}(1-W) Y_{t}, i$ 恒成立, 达到非线性模型到 线性模型的转换的目的。

线性化目标函数为

$$
\min T^{E C}=
$$

$\sum_{k \in K} \sum_{t=1}^{p_{k}-1}\left(\left(U_{k, t+1}-V_{k, t}-\sum_{i \in l} \sum_{h \in H_{i}} \sum_{j \in J_{i, h}}\left(p_{i, h, j, k}^{t} A_{i, h, j, k, t}\right)\right) P_{i d l e}^{k}+E_{n m m}^{k} W_{k, t}\right)+$ $\sum_{i \in I} \sum_{h \in H_{i}} \sum_{j \in J_{i, h}} \sum_{k \in K_{i, h, j}} \sum_{t \in L_{k}} P_{i, h, j, k} p_{i, h, j, k}^{t} Y_{i, h, j, k, t}+P_{0} C_{\max }$

\subsection{3 约束条件}

表 2 给出了模型 1 所需要的所有约束方程。

表 2 模型 1 所有约束条件

\begin{tabular}{cc||cc}
\hline 编号 & 约束 & 编号 & 约束 \\
\hline 1 & $(11)$ & 14 & $(24)$ \\
2 & $(12)$ & 15 & $(25)$ \\
3 & $(13)$ & 16 & $(26)$ \\
4 & $(14)$ & 17 & $(27)$ \\
5 & $(15)$ & 18 & $(28)$ \\
6 & $(16)$ & 19 & $(29)$ \\
7 & $(17)$ & 20 & $(30)$ \\
8 & $(18)$ & 21 & $(31)$ \\
9 & $(19)$ & 22 & $(32)$ \\
10 & $(20)$ & 23 & $(33)$ \\
11 & $(21)$ & 24 & $(34)$ \\
12 & $(22)$ & 25 & $(35)$ \\
13 & $(23)$ & 26 & $(36)$ \\
\hline \multicolumn{5}{|c}{} \\
\end{tabular}

$\sum_{k \in K_{i, h, j}} \sum_{t \in L_{k}} Y_{i, h, j, k, t}=X_{i, h}, \forall i \in I, h \in H_{i}, j \in J_{i, h}$

$$
\begin{gathered}
\sum_{i \in I} \sum_{h \in H_{i}} \sum_{j \in J_{i, h}} Y_{i, h, j, k, t} \leqslant 1, \forall k \in K, t \in L_{k} \\
B_{i, h, j} \leqslant M X_{i, h}, \forall i \in I, h \in H_{i}, j \in J_{i, h} \\
\sum_{i \in I} \sum_{h \in H_{i}} \sum_{j \in J_{i, h}} Y_{i, h, j, k, t} \geqslant \sum_{i \in I} \sum_{h \in H_{i}} \sum_{j \in J_{i, h}} Y_{i, h, j, k, t+1}, \\
\forall k \in K, t \in\left\{1,2, \cdots, p_{k}-1\right\} \\
S_{k, t} \leqslant B_{i, j}+M\left(1-Y_{i, h, j, k, t}\right), \\
\forall i \in I, h \in H_{i}, j \in J_{i, h}, k \in K_{i, h, j}, t \in L_{k} \\
S_{k, t}+M\left(1-Y_{i, h, j, k, t}\right) \geqslant B_{i, j},
\end{gathered}
$$


$\forall i \in I, h \in H_{i}, j \in J_{i, h}, k \in K_{i, h, j}, t \in L_{k}$

$$
B_{i, h, j j} \geqslant B_{i, h, j}+\sum_{k \in K_{i, h, j}} \sum_{t \in L_{k}} p_{i, h, j, k}^{t} Y_{i, h, j, k, t}
$$

$\forall i \in I, h \in H_{i}, j \in J_{i, h}, j j \in J_{i, h}, j<j j, Q_{i, h, j, j j}=1$

$$
B_{i, h, j j} \geqslant B_{i, h, j}+\sum_{k \in K_{i, h, j}} \sum_{t \in L_{k}} p_{i, h, j, k}^{t} Y_{i, h, j, k, t}-M\left(1-Z_{i, j, j j}\right)
$$$$
\forall i \in I, h \in H_{i}, j \in J_{i, h}, j j \in J_{i, h}, j<j j, Q_{i, h, j, j j}=2
$$

$$
B_{i, h, j} \geqslant B_{i, h, j j}+\sum_{k \in K_{i, h, i j}} \sum_{t \in L_{k}} p_{i, h, j j, k}^{t} Y_{i, h, j j, k, t}-M Z_{i, j, j j}
$$

$\forall i \in I, h \in H_{i}, j \in J_{i, h}, j j \in J_{i, h}, j<j j, Q_{i, h, j, j j}=2$

$$
B_{i, h, j}+\sum_{k \in K_{i, h, j}} \sum_{t \in L_{k}} p_{i, h, j, k}^{t} Y_{i, h, j, k, t} \leqslant C_{\max },
$$

$\forall i \in I, h \in H_{i}, j \in J_{i, h}$

$$
\sum_{t=1}^{p_{k}-1} W_{k, t} \leqslant N_{k}, \forall k \in K
$$

$B_{i, h, j}, S_{k, t} \geqslant 0, \forall i \in I, h \in H_{i}, j \in J_{i, h}, k \in K_{i, h, j}, t \in L_{k}$

$$
S_{k, t+1}-S_{k, t}-\sum_{i \in I} \sum_{h \in H_{i}} \sum_{j \in J_{i, h}} p_{i, h, j, k}^{t} Y_{i, h, j, k, t} \leqslant T_{k}^{B}+M W_{k, t}
$$$$
\forall k \in K, t \in\left\{1,2, \cdots, p_{k}-1\right\}
$$

$$
S_{k, t+1}-S_{k, t}-\sum_{i \in I} \sum_{h \in H_{i}} \sum_{j \in J_{i, h}} p_{i, h, j, k}^{t} Y_{i, h, j, k, t} \geqslant T_{k}^{B} W_{k, t},
$$$$
\forall k \in K, t \in\left\{1,2, \cdots, p_{k}-1\right\}
$$

$U_{k, t+1} \geqslant S_{k, t+1}-M W_{k, t}, \forall k \in K, t \in\left\{1,2, \cdots, p_{k}-1\right\}$

$$
U_{k, t+1} \leqslant S_{k, t+1}+M W_{k, t}, \forall k \in K, t \in\left\{1,2, \cdots, p_{k}-1\right\}
$$

$$
\begin{gathered}
U_{k, t+1} \leqslant M\left(1-W_{k, t}\right), \forall k \in K, t \in\left\{1,2, \cdots, p_{k}-1\right\} \\
U_{k, t} \geqslant 0, \forall k \in K, t \in L_{k} \\
V_{k, t} \geqslant S_{k, t}-M W_{k, t}, \forall k \in K, t \in\left\{1,2, \cdots, p_{k}-1\right\} \\
V_{k, t} \leqslant S_{k, t}+M W_{k, t}, \forall k \in K, t \in\left\{1,2, \cdots, p_{k}-1\right\} \\
V_{k, t} \leqslant M\left(1-W_{k, t}\right), \forall k \in K, t \in\left\{1,2, \cdots, p_{k}-1\right\} \\
V_{k, t} \geqslant 0, \forall k \in K, t \in\left\{1,2, \cdots, p_{k}-1\right\} \\
A_{i, h, j, k, t} \leqslant Y_{i, h, j, k, t}
\end{gathered}
$$$$
\forall i \in I, h \in H_{i}, j \in J_{i, h}, k \in K, t \in\left\{1, \cdots, p_{k}-1\right\}
$$

$$
A_{i, h, j, k, t} \leqslant 1-W_{k, t}
$$

$\forall i \in I, h \in H_{i}, j \in J_{i, h}, k \in K, t \in\left\{1, \cdots, p_{k}-1\right\}$

$$
A_{i, h, j, k, t} \geqslant Y_{i, h, j, k, t}-W_{k, t}
$$

$\forall i \in I, h \in H_{i}, j \in J_{i, h}, k \in K, t \in\left\{1, \cdots, p_{k}-1\right\}$

式(11)表示任一工件只能选择一种加工组合。式(12) 表示两决策变量之间的关系。式(13)表示任一机器 的任一位置最多安排一个工序。式(14)用于约束未 被选中组合中工序的开始时间为 0 。式(15)表示任一
机器的位置按照先后顺序安排工件。式(16)、(17) 表示机器位置的开始时间等于其所加工工序的开 工时间。式(18)用于保证同一工件有先后约束的工 序按照先后顺序加工。式(19)、(20)约束同一工件 没有先后约束的工序之间必须且只能确定一种先 后关系。式(21)为最大完工时间约束。式(22)为机 床中途最大关机重启次数约束。式(24)、(25)为机 床位置关机重启策略约束，当机床 $k$ 的 $t$ 到 $t+1$ 位置间存在关机重启策略时, 即 $W_{k, t}=1$, 则第 $t+1$ 位置的开始时间 $S_{k, t+1}$ 与第 $t$ 位置的结束时间 $S_{k, t}+\sum_{i \in I} \sum_{h \in H_{i}} \sum_{j \in J_{i, h}} p_{i, h, j, k}^{t} Y_{i, h, j, k, t}$ 之差必定大于机床 $k$ 的 空载平衡时间 $T_{k}^{B}$; 否则, $W_{k, t}=0$, 不实行关机重 启策略。式(25)同时保证了任一机器位置的开始时 间不小于其紧前位置的结束时间。式(26) (29)保证 $U_{k, t+1}=\left(1-W_{k, t}\right) S_{k, t+1}$ 恒成立, 当 $W_{k, t}=0$ 时，由式(26) 可知 $U_{k,+1} \geqslant S_{k, t+}$; 由 (27) 可知, $U_{k, t+1} \leqslant S_{k, t+1}$, $U_{k, t+1}=S_{k, t+1}$ 。因此, 式(26)、(27)保证了当 $W_{k, t}=0$ 时, $U_{k, t+1}=\left(1-W_{k, t}\right) S_{k \neq t}$ 成立。当 $W_{k, t}=1$ 时, 由式(28)、 (29) 可知, $U_{k, t+1}=0$, 保证了 $U_{k, t+1}=\left(1-W_{k, t}\right) S_{k, t+1}$ 成 立。式(30) (33) 保证 $V_{k, t}=\left(1-W_{k, t}\right) S_{k, t}$ 恒成立, 其 中, 式 (30)、(31) 保证当 $W_{k, t}=0$ 时, $\left.V_{k,}=S_{, k} \neq_{t} 1-W,\right)_{k}$ 成立, 式(32)、(33)保证当 $W_{k, t}=1$ 时, $V_{k, t}=0=\left(1-W_{k, t}\right) S_{k, t}$ 成立。式(34)、(35) 保证 $A_{i, h, j, k, t}=\left(1-W_{k, t}\right) Y_{i, h, j, k, t}$ 恒成立, 其中, 式(34)、

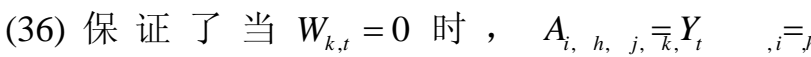
$\left(1-W_{k, t}\right) Y_{i, h, j, k, t}$ 成立, 式 (35) 保证当 $W_{k, t}=1$ 时, $A_{i, h, j, k, \bar{t}} 0=\left(1-W_{k}\right) Y_{, i, h}$ 成立。

\section{2 模型 2}

与模型 1 相比, 模型 2 添加连续决策变量 $F_{k, t}$, 表示机器 $k$ 上第 $t$ 个位置的结束时间, 虽然目标函数 (式(37))仍然为非线性的, 但是只存在两个非线性项 $\left(1-W_{k, t}\right) S_{k, t+1}$ 与 $\left(1-W_{k, t}\right) F_{k, t}$, 与模型 1 比起来, 只 需要引入两个中间连续决策变量 $U_{k, t}$ 与 $V V_{k, t}$, 其中 $U_{k, t}$ 与模型 1 中含义相同，用 $U_{k, t+1}$ 来代替非线性项 $\left(1-W_{k, t}\right) S_{k, t+1}$, 中间决策变量 $V V_{k, t}$ 用来代替非线性 项 $\left(1-W_{k, t}\right) F_{k, t}$, 实现非线性目标函数(式(37)) 到线性 目标函数(式(38))的转换。

\subsection{1 决策变量}

$X_{i, h} 、 Y_{i, h, j, k, t} 、 Z_{i, j, j j} 、 W_{k, t} 、 B_{i, j} 、 S_{k, t} 、 F_{k, t} 、 U_{k, t} 、$ $C_{\max }$ 含义与模型 1 相应参数含义相同。

$V V_{k, t}$ ：中间连续决策变量，用来代替非线性项 $\left(1-W_{k, t}\right) F_{k, t}$ 。

\subsection{2 目标函数}

$$
\min T^{E C}=
$$


$\sum_{k \in K} \sum_{t=1}^{p_{k}-1}\left(\left(1-W_{k, t}\right)\left(S_{k, t+1}-F_{k, t}\right) P_{i d l e}^{k}+E_{\text {turn }}^{k} W_{k, t}\right)+$

$\sum_{i \in I} \sum_{h \in H_{i}} \sum_{j \in J_{i, h}} \sum_{k \in K_{i, h, j}} \sum_{t \in L_{k}} P_{i, h, j, k} p_{i, h, j, k}^{t} Y_{i, h, j, k, t}+P_{0} C_{\max }$

线性化目标函数为

$\min T^{E C}=\sum_{k \in K} \sum_{t=1}^{p_{k}-1}\left(\left(U_{k, t+1}-V V_{k, t}\right) P_{i d l e}^{k}+E_{\text {turn }}^{k} W_{k, t}\right)+$

$\sum_{i \in I} \sum_{h \in H_{i}} \sum_{j \in J_{i, h}} \sum_{k \in K_{i, h, j}} \sum_{t \in L_{k}} P_{i, h, j, k} p_{i, h, j, k}^{t} Y_{i, h, j, k, t}+P_{0} C_{\max }$

\subsection{3 约束条件}

表 3 给出了模型 2 所需要的所有约束方程。

表 3 模型 2 所有约束条件

\begin{tabular}{cc||cc}
\hline 编号 & 约束 & 编号 & 约束 \\
\hline 1 & $(11)$ & 13 & $(23)$ \\
2 & $(12)$ & 14 & $(26)$ \\
3 & $(13)$ & 15 & $(27)$ \\
4 & $(14)$ & 16 & $(28)$ \\
5 & $(15)$ & 17 & $(29)$ \\
6 & $(16)$ & 18 & $(39)$ \\
7 & $(17)$ & 19 & $(40)$ \\
8 & $(18)$ & 20 & $(41)$ \\
9 & $(19)$ & 21 & $(42)$ \\
10 & $(20)$ & 22 & $(43)$ \\
11 & $(21)$ & 23 & $(44)$ \\
12 & $(22)$ & 24 & $(45)$ \\
\hline
\end{tabular}

$F_{k, t}=S_{k, t}+\sum_{i \in I} \sum_{h \in H_{i}} \sum_{j \in J_{i, h}} p_{i, h, j, k}^{t} Y_{i, h, j, k, t}, \forall k \in K, t \in L_{k}$

$S_{k, t+1}-F_{k, t} \leqslant T_{k}^{B}+M W_{k, t}, \forall k \in K, t \in\left\{1,2, \cdots, p_{k}-1\right\}$

$S_{k, t+1}-F_{k, t} \geqslant T_{k}^{B} W_{k, t}, \forall k \in K, t \in\left\{1,2, \cdots, p_{k}-1\right\}$

$V V_{k, t} \geqslant F_{k, t}-M W_{k, t}, \forall k \in K, t \in\left\{1,2, \cdots, p_{k}-1\right\}$

$V V_{k, t} \leqslant F_{k, t}+M W_{k, t}, \forall k \in K, t \in\left\{1,2, \cdots, p_{k}-1\right\}$

$V V_{k, t} \leqslant M\left(1-W_{k, t}\right), \forall k \in K, t \in\left\{1,2, \cdots, p_{k}-1\right\}$

$$
V V_{k, t} \geqslant 0, \forall k \in K, t \in\left\{1,2, \cdots, p_{k}-1\right\}
$$

式(39)表示机床位置结束时间等于机床位置开始时 间加上安排在该位置工序的加工时间; 式(40)、(41) 分别与式(24)、(25)作用形同; 式(42) (45)用来保 证 $V V_{k, t}=\left(1-W_{k, t}\right) F_{k, t}$ 恒成立, 其中式(42)、(43)用来 保证当 $W_{k, t}=0$ 时, $V V_{k, t}=F_{k, t}=\left(1-W_{k t}\right) F_{k t}$ 成立, 式 (44)、(45)保证当 $W_{k, t}=1$ 时, $V V_{k, t}=0=\left(1-W_{k, t}\right) F_{k, t}$ 成立。

\section{3 模型 3}

不同于模型 1 和模型 2 , 模型 3 基于空闲能耗 的建模方法, 直接定义空闲段能耗 $E_{k, t}$ 决策变量, 目标函数(46)本身则为线性的, 因此不需要引入如
模型 1 与模型 2 中的中间决策变量以及约束方程等。 2.3.1 决策变量

$X_{i, h} 、 Y_{i, h, j, k, t} 、 Z_{i, j, j j} 、 W_{k, t} 、 B_{i, j} 、 S_{k, t}$ 以及 $C_{\text {max }}$ 含 义与模型 1 相应参数含义相同。

$E_{k, t}$ : 机床 $k$ 第 $t$ 到 $t+1$ 位置间所需要消耗的能 量(待机或者关机重启)。

2.3.2 目标函数

$$
\min \sum_{k \in K} \sum_{t=1}^{p_{k}-1} E_{k, t}+
$$

$$
\sum_{i \in I} \sum_{h \in H_{i}} \sum_{j \in J_{i, h}} \sum_{k \in K_{i, h, j}} \sum_{i \in L_{k}} P_{i, h, j, k} p_{i, h, j, k}^{t} Y_{i, h, j, k, t}+P_{0} C_{\max }
$$

式中, 第一项表示机床空闲等待能耗加上关机重启所 需要的能耗, 第二项为加工能耗, 第三项为公共能耗。 2.3.3 约束条件

表 4 给出了模型 3 所有约束条件。

表 4 模型 3 所有约束条件

\begin{tabular}{cc||cc}
\hline 编号 & 约束 & 编号 & 约束 \\
\hline 1 & $(11)$ & 9 & $(19)$ \\
2 & $(12)$ & 10 & $(20)$ \\
3 & $(13)$ & 11 & $(21)$ \\
4 & $(14)$ & 12 & $(22)$ \\
5 & $(15)$ & 13 & $(23)$ \\
6 & $(16)$ & 14 & $(25)$ \\
7 & $(17)$ & 15 & $(47)$ \\
8 & $(18)$ & 16 & $(48)$ \\
\hline
\end{tabular}

$$
\begin{gathered}
E_{k, t} \geqslant E_{u m m}^{k} W_{k, t}, \forall k \in K, t \in\left\{1,2, \cdots, p_{k}-1\right\} \\
E_{k, t} \geqslant\left(S_{k, t+1}-S_{k, t}-\sum_{i \in I} \sum_{h \in H_{i}} \sum_{j \in J_{i, h}} p_{i, h, j, k}^{t} Y_{i, h, j, k, t}\right) P_{i d l e}^{k}-M W_{k, t} \\
\forall k \in K, t \in\left\{1,2, \cdots, p_{k}-1\right\}
\end{gathered}
$$

成对约束(47)与(48)表示, 当机床 $k$ 的 $t$ 到 $t+1$ 位置 间存在关机重启策略时, 即 $W_{k, t}=1$, 机床 $k$ 的 $t$ 到 $t+1$ 位置间的能耗不小于机床 $k$ 的关机以及重启一 次所需要的能耗，否则当机床 $k$ 的 $t$ 到 $t+1$ 位置间不 存在关机重启策略时, 即 $Z_{k, t}=0$, 机床 $k$ 的 $t$ 到 $t+1$ 位置间的能耗由具体待机时间决定。该模型中的约 束(25)用来保证当机床 $k$ 的 $t$ 到 $t+1$ 位置间存在关 机重启策略时, 即 $W_{k, t}=1$, 机床 $k$ 的 $t$ 到 $t+1$ 位置 间的空闲时间不小于机床 $k$ 的空载平衡时间 $T_{k}^{B}$, 以 满足关机重启策略的时间要求。

\section{3 模型对比}

本节从尺寸复杂度与计算复杂度对 3 个 MILP 模型进行比较, 尺寸复杂度从 0-1 变量个数、约束 数目以及连续决策变量个数 3 个方面进行对比。计 
算复杂度从在规定时间内可求最优解总数 (Total), 包括 $g a p=0$ 最优解个数 (Total0) 与 $g a p \neq 0$ 最优解个数 (Total1). 当 Total 相同时, 对比 Total0, 当 Total 与 Total 1 都相同时, 对比 Total1、Total、Total0 与 Total1 越大, 模型越好。当 Total、Total0 与 Total 1 都相同 时, 求解时间 Time 也是一个重要评价指标, Time 越 小越好。gap 表示目标函数值的容差, 可定义为 $|C S-B S| /|C S| \times * \%$, 其中 $C S$ 表示至目前可以找到的 最优解, $B S$ 表示可能的最优解, 是当前所有解的 下限。可见, gap 值越小越好, 当 gap=0 时, 则得 到问题的最优解, 程序会自动停止。因此, gap 值 可以作为评价 MILP 模型求解性能的一个评价指标 或者停止条件。
图 2 为工件的柔性工艺路线网络图。由图 2 可 以看出, 工件 1 有 3 个加工组合(1-2-5,1-3-5,1-4-5), 其中工序 1 与 5 是所有组合中共有的工序; 工件 2 只有一个加工组合，其中工序 2、3、4 之间没有先 后顺序约束; 工件 3 有两个加工组合 (1-2-3-6-7,1-4-5-6-7), 其中工序 $1 、 6$ 以及 7 为公共 工序; 工件 4 只有 1 个加工组合，其中工序 3、4 与工序 $5 、 6$ 之间没有优先约束; 工件 5 有两种加工 组 合 (1-2-3-4-5-6-7-8-9-10-11-14,1-2-3-4-5-6-7-8-1213-14), 其中工序 $1 、 2 、 3 、 4 、 5 、 6 、 7 、 8$ 以及 14 为公共工序，其中工序 $2 、 3$ 与工序 $4 、 5$ 没有优先 关系，工序 $1 、 2 、 3 、 4 、 5 、 6$ 与工序 $7 、 8 、 9 、 10 、$ $11 、 12 、 13 、 14$ 也没有优先关系。

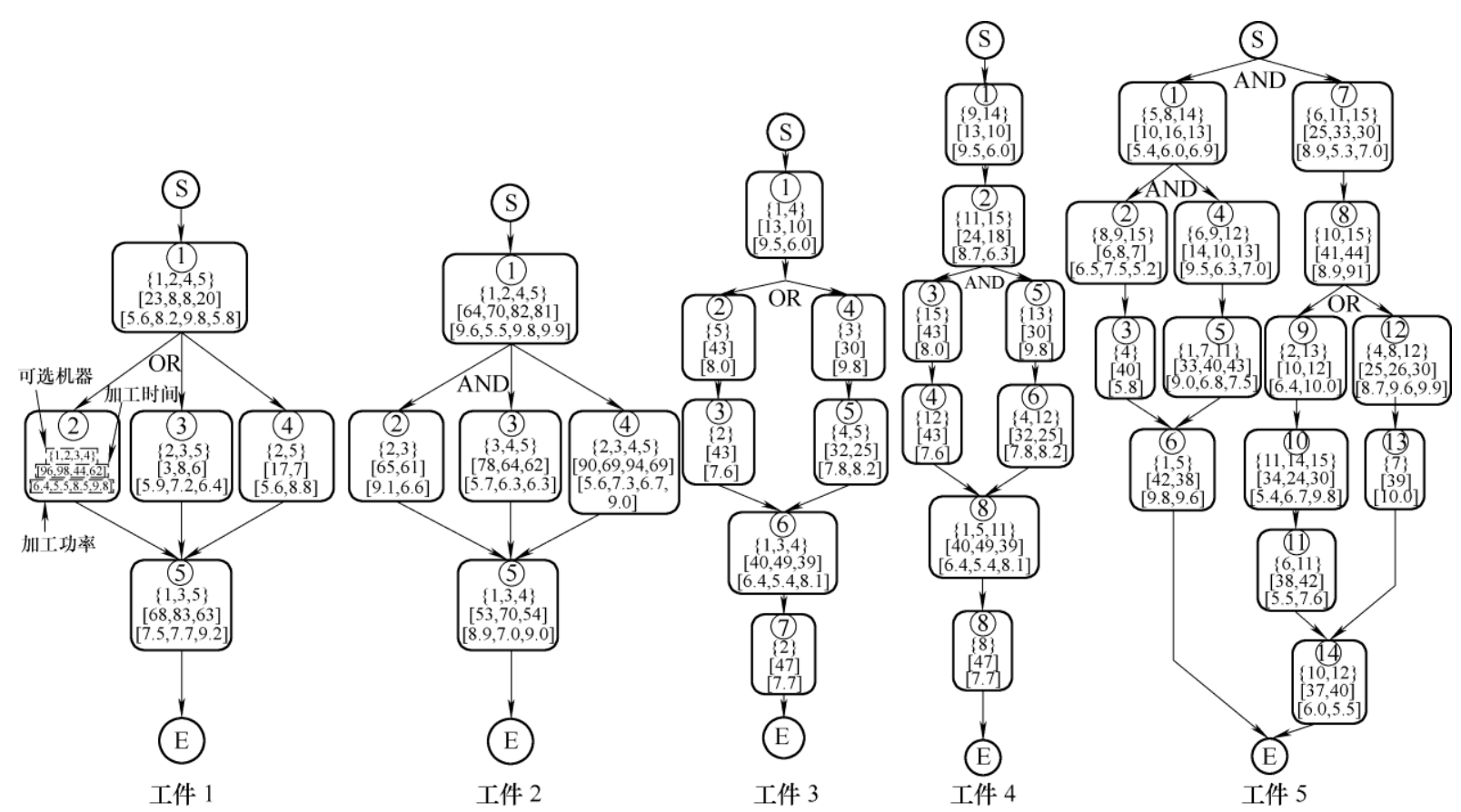

图 2 工件柔性工艺路线网络图

为了得到不同规模的测试实例, 分别取工件 1 与 2 , 工件 1 与 3 , 工件 2 与 3 , 工件 $1 、 2$ 以及 3 , 工件 5 , 工件 4 与 5,2 倍工件 1 与 2,2 倍工件 1 与 3,2 倍工件 2 与 3 以及 2 倍工件 $1 、 2 、 3$, 总共得 到 10 个规模不同的实例。表 5 中, $1+2$ 表示工件 1 、 2 组合, $(1+2) * 2$ 表示工件 1、2 都取两个的组合, 其他含义相同。设所有机床允许中途关机重启次数 $N_{k}$ 为 5 , 公共功率 $P_{0}$ 为 20 。其他具体加工时间、 加工功率以及机床能耗信息见表 5 与图 2 与图 3。

本文 3 个 MILP 模型都由 IBM ILOG CPLEX12.7.1
求解, 编程语言使用其自带 OPL 语言。10 组测试 实例都在 Dell Vostro 3900 台式机上进行求解, 该台 式机配有 i5-4460 3.20GHz 双核四线程 CPU、8G 运 行内存。

\section{1 模型尺寸复杂度对比}

由表 6 以及图 3 可以看出, 模型 2 与模型 3 的 $0-1$ 决策变量数相等且比模型 1 少。这是因为模型 2 与 3 只含有 $X_{i, h} 、 Y_{i, h, j, k, t} 、 Z_{i, j, j j}$ 以及 $W_{k, t} 4$ 个 0-1 决 策变量, 模型 1 除了这 4 个决策变量外, 还引入了 中间 0-1 决策变量 $A_{i, h, j, k, t}$ 。

表 5 机床能耗数据

\begin{tabular}{cccccccccccccccc}
\hline 能耗 & M1 & M2 & M3 & M4 & M5 & M6 & M7 & M8 & M9 & M10 & M11 & M12 & M13 & M14 & M15 \\
\hline$T^{B}$ & 10 & 15 & 20 & 10 & 15 & 20 & 10 & 15 & 20 & 10 & 15 & 20 & 10 & 15 & 20 \\
$E_{\text {uum }}$ & 10 & 30 & 60 & 10 & 30 & 60 & 10 & 30 & 60 & 10 & 30 & 60 & 10 & 30 & 60 \\
$P_{\text {idle }}$ & 1 & 2 & 3 & 1 & 2 & 3 & 1 & 2 & 3 & 1 & 2 & 3 & 1 & 2 & 3 \\
\hline
\end{tabular}



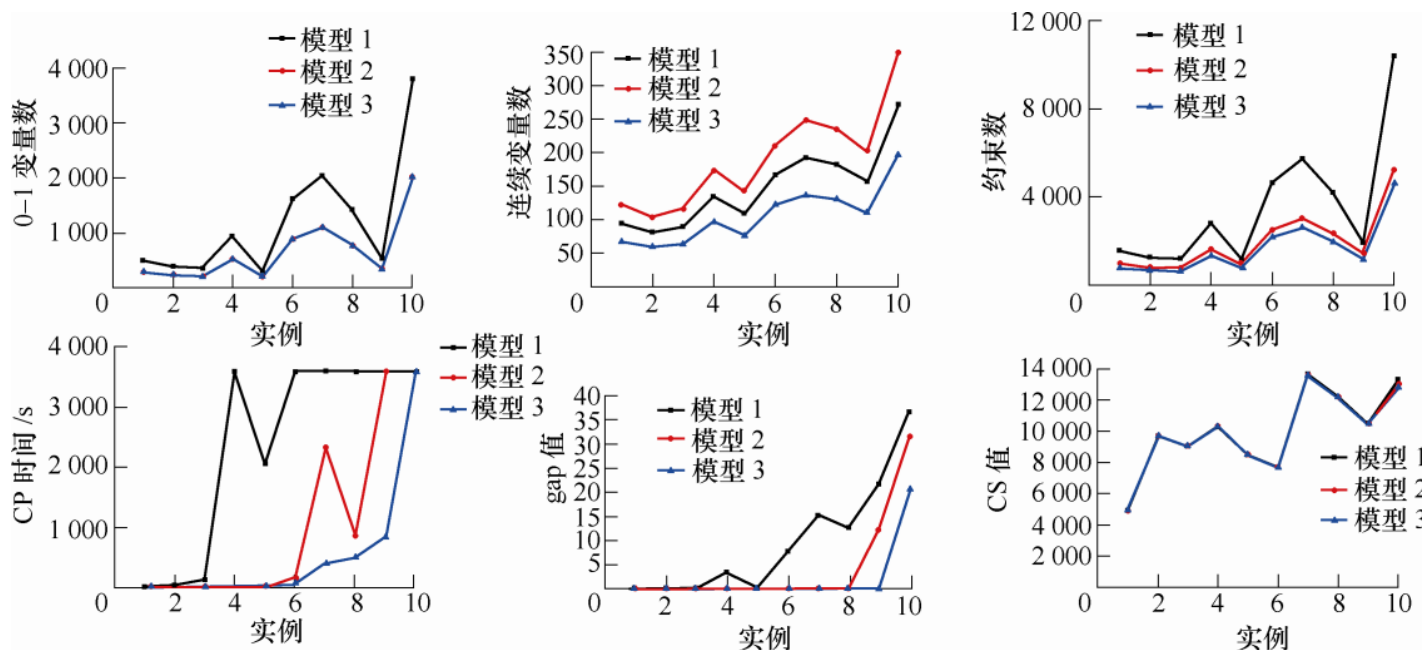

- - 模型 1
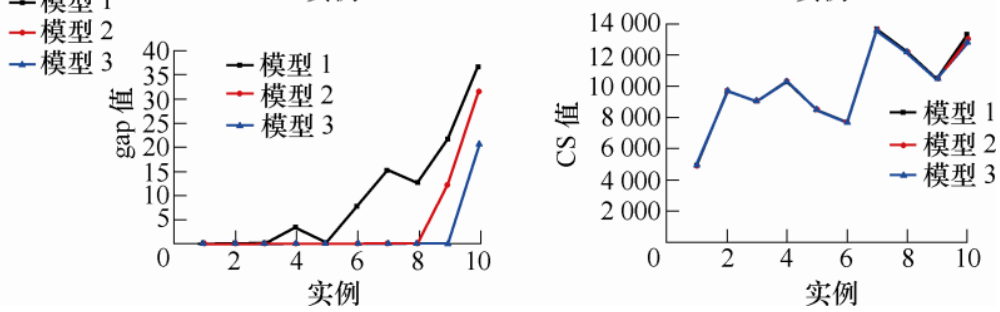

图 33 个 MILP 模型尺寸复杂度以及计算复杂度对比图

表 63 个 MILP 模型尺寸复杂度比较

\begin{tabular}{|c|c|c|c|c|c|c|c|c|c|c|}
\hline \multirow{2}{*}{ 实例 } & \multirow{2}{*}{ 工件 } & \multicolumn{3}{|c|}{ 模型 1} & \multicolumn{3}{|c|}{ 模型 2} & \multicolumn{3}{|c|}{ 模型 3} \\
\hline & & $0-1$ & 约束 & 连续 & $0-1$ & 约束 & 连续 & $0-1$ & 约束 & 连续 \\
\hline 1 & $1+3$ & 391 & 1247 & 81 & 230 & 803 & 103 & 230 & 657 & 59 \\
\hline 2 & $2+3$ & 352 & 1184 & 89 & 206 & 793 & 115 & 206 & 615 & 63 \\
\hline 3 & $1+2$ & 500 & 1551 & 94 & 288 & 966 & 122 & 288 & 772 & 66 \\
\hline 4 & $1+2+3$ & 937 & 2796 & 134 & 521 & 1619 & 172 & 521 & 1345 & 96 \\
\hline 5 & 5 & 304 & 1172 & 109 & 210 & 942 & 142 & 210 & 762 & 76 \\
\hline 6 & $(1+3) \times 2$ & 1619 & 4619 & 166 & 881 & 2488 & 210 & 881 & 2166 & 122 \\
\hline 7 & $(2+3) \times 2$ & 1425 & 4203 & 182 & 775 & 2352 & 234 & 775 & 1966 & 130 \\
\hline 8 & $(1+2) \times 2$ & 2037 & 5727 & 192 & 1097 & 3014 & 248 & 1097 & 2596 & 136 \\
\hline 9 & $4+5$ & 539 & 1942 & 156 & 343 & 1432 & 202 & 343 & 1148 & 110 \\
\hline 10 & $(1+2+3) \times 2$ & 3795 & 10427 & 272 & 2005 & 5204 & 348 & 2005 & 4626 & 196 \\
\hline
\end{tabular}

在约束数量方面, 由表 6 及图 3 可以看出, 从 多到少依次为模型 1 、模型 2 、模型 3 。模型 3 基于 空闲能耗的建模思想, 不需要引入中间决策变量以 及相关的约束, 因此模型 3 约束最少。模型 1 虽然 比模型 2 少了决策变量 $F_{k, t}$, 但是需要比模型 2 多 引入中间决策变量 $A_{i, h, j, k, t}$ 以及相关约束, 因此模型 1 比模型 2 约束多。

在连续决策变量方面, 模型 3 同样是最少, 只含有 $B_{i, j} 、 S_{k, t} 、 E_{k, t}$ 以及 $C_{\max } 4$ 个连续决策变 量; 模型 2 最多, 含有 $B_{i, j} 、 S_{k, t} 、 F_{k, t} 、 C_{\text {max }} 、 U_{k, t}$ 以及 $V V_{k, t} 6$ 个连续决策变量; 模型 1 含有 5 个连 续决策变量, 分别为 $B_{i, j} 、 S_{k, t} 、 C_{\text {max }} 、 U_{k, t}$ 以及 $V_{k, t}$ 。

\section{2 模型计算复杂度对比}

3 个 MILP 模型针对 10 个实例的求解结果见表 7 。可以看出, 模型 3 在规定 $3600 \mathrm{~s}$ 内可以求出 10 个实例中 9 个最优解 $(T o t a l 0=9)$, 模型 2 可以得到 8 个最优解 $($ Total $=8)$, 其中 7 个 gap=0 最优解
$($ Total $0=7)$, 对于实例 (工件 4+5), 虽然在 $3600 \mathrm{~s}$ 内 可以得到最优解但是不能证明所得解为最优解 ( gap $=12.22 \%)$ 。模型 1 求解效果最差, 在 $3600 \mathrm{~s}$ 只 能得到 8 个实例的最优解(Total $=8$ ), 其中 4 个 gap $=0$ 最优解 $($ Total $0=4), 4$ 个 gap $\neq 0$ 最优解 $($ Total $1=1)$ 。从 求解时间上可以看出, 模型 3 所需总时间远小于模 型 1 与模型 2 。从 $3600 \mathrm{~s}$ 内可得到的最优解 $(C S)$ 来 看, 模型 3 所得到的解是 3 个模型中最小的。同样 从 gap 值可以看出, 模型 3 是 3 个模型中最小的。 究其原因, 模型 3 基于空闲能耗的建模方法, 尺寸 复杂度最小，具有最少的 0-1 决策变量、约束方程 以及连续决策变量数, 模型 3 求解效果远好于模型 1 与模型 2 , 尤其针对大规模实例。模型 1 比模型 2 多引入中间决策变量 $A_{i, h, j, k, t}$ 以及相关约束, 因此使 模型 1 变得复杂，在 $0-1$ 决策变量数以及约束数方 面, 模型 1 都多于模型 2 , 因此, 模型 1 效果比模 型 2 差。

图 4 给出了实例 9 的最优解调度甘特图。 
表 73 个 MILP 模型计算复杂度比较

\begin{tabular}{|c|c|c|c|c|c|c|c|c|c|}
\hline \multirow{2}{*}{ 实例 } & \multicolumn{3}{|c|}{ 模型 1} & \multicolumn{3}{|c|}{ 模型 2} & \multicolumn{3}{|c|}{ 模型 3} \\
\hline & $C S$ & Time/s & gap & $C S$ & Time/s & gap & $C S$ & Time/s & gap \\
\hline 1 & 4902.1 & 17.33 & 0 & 4902.1 & 0.17 & 0 & 4902.1 & 0.16 & 0 \\
\hline 2 & 9660.5 & 39.69 & 0 & 9660.5 & 0.50 & 0 & 9660.5 & 1.23 & 0 \\
\hline 3 & 9046.9 & 127.17 & 0 & 9046.9 & 0.66 & 0 & 9046.9 & 1.33 & 0 \\
\hline 4 & 10253.8 & 3600 & 3.26 & 10253.8 & 2.93 & 0 & 10253.8 & 8.50 & 0 \\
\hline 5 & 8477.5 & 2048.31 & 0 & 8477.5 & 6.85 & 0 & 8477.5 & 10.19 & 0 \\
\hline 6 & 7668.6 & 3600 & 7.92 & 7668.6 & 158.62 & 0 & 7668.6 & 64.23 & 0 \\
\hline 7 & $13669.5^{*}$ & 3600 & 15.13 & 13394.6 & 2325.15 & 0 & 13394.6 & 394.53 & 0 \\
\hline 8 & 12164.8 & 3600 & 12.64 & 12164.8 & 856.43 & 0 & 12164.8 & 495.38 & 0 \\
\hline 9 & 10448.6 & 3600 & 21.62 & 10448.6 & 3600 & 12.22 & 10448.6 & 853.95 & 0 \\
\hline 10 & $13287.7 *$ & 3600 & 36.58 & $13011.3^{*}$ & 3600 & 31.45 & $12763.9^{*}$ & 3600 & 20.57 \\
\hline 总和 & 99580 & 23832.5 & 97.15 & 99028.7 & 10551.31 & 43.67 & 98781.3 & 5429.5 & 20.57 \\
\hline Total0 & & 4 & & & 8 & & & 9 & \\
\hline Total 1 & & 4 & & & 1 & & & 0 & \\
\hline Total & & 8 & & & 9 & & & 9 & \\
\hline
\end{tabular}

注: *的解表示此解为可行解但非最优解。

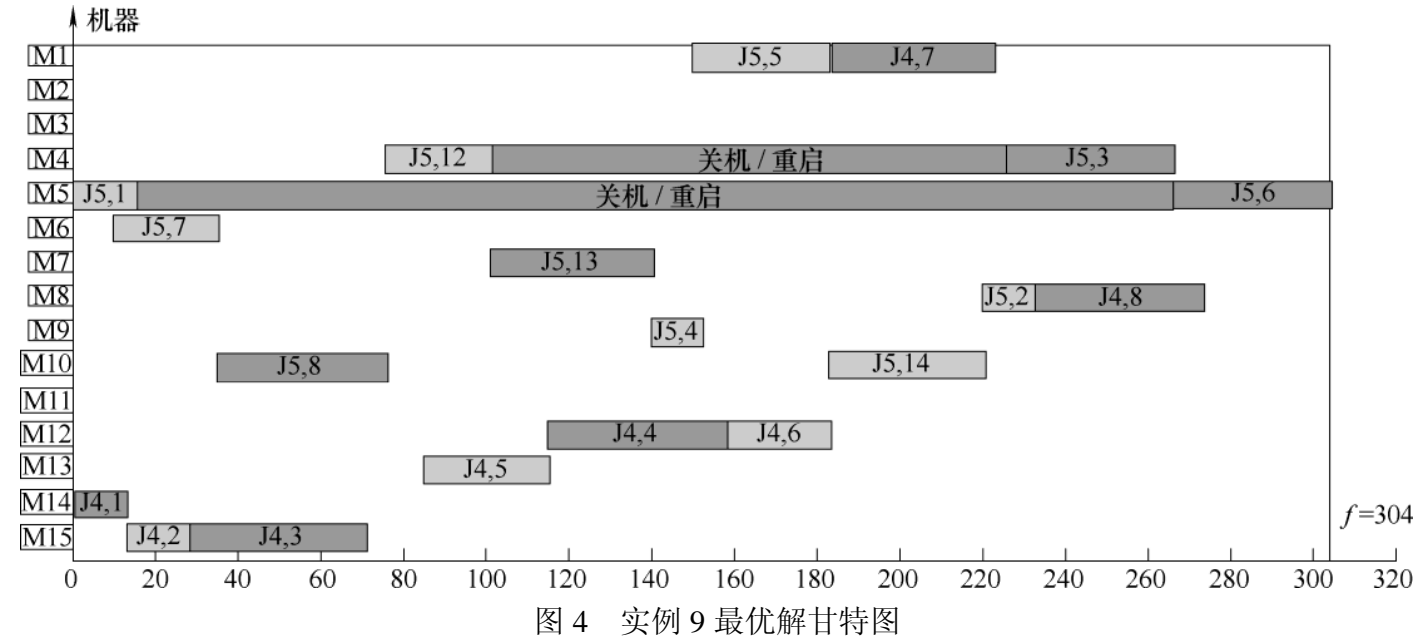

\section{4 结论}

本文研究面向节能的工艺规划与调度集成问 题，考虑了基于不同的建模思路并同时考虑关机 重启节能策略, 建立了 3 个 MILP 模型, 通过使用 CPLEX 求解器对具体实例的求解验证了本文模型 的有效性。同时, 对 3 个 MILP 模型从建模过程、 尺寸复杂度、计算复杂度等方面进行了详细的对比 分析, 试验结果表明基于空闲能耗的 MILP 模型建 模过程简单, 尺寸复杂度与计算复杂度都好于基于 空闲时间的两个 MILP 模型。在今后实际应用时, 应优先考虑基于空闲能耗建模思想的模型 3 。

MILP 模型的求解主要局限于计算机性能以及 相关求解软件, 随着计算机性能的日益提升以及求 解软件(CPLEX、GROUBI)的逐步完善, 针对调度 问题 MILP 建模的研究将会引起越来越多学者的关
注。车间中的能耗设备还有很多，如叉车、吊车等 的运输能耗, 下一步研究工作将考虑更多的实际情 况, 如把运输能耗、转换能耗、换刀能耗、峰值功 率约束、分时电价等考虑进来，构建相应的 IPPS 问题的 MILP 模型。进一步结合元启发式算法，探 索针对能耗目标有效的解码方法、分配规则、节能 策略等，探索求解中大规模问题的混合求解方法。

\section{参 考 文 献}

[1] IEA. Tracking industrial energy efficiency and $\mathrm{CO}_{2}$ emissions[R]. Paris: OECD/IEA: 2007.

[2] 刘飞, 王秋莲, 刘高君. 机械加工系统能量效率研究的 内容体系及发展趋势[J]. 机械工程学报, 2013，49(19): 87-94.

LIU Fei, WANG Qiulian, LIU Gaojun. Content architecture and future trends of energy efficiency research on machining systems[J]. Journal of Mechanical Engineering, 2013, 49(19): 87-94. 
[3] MOUZON G, YILDIRIM M B, TWOMEY J. Operational methods for minimization of energy consumption of manufacturing equipment[J]. International Journal of Production Research, 2007, 45(18-19): 4247-4271.

[4] GUTOWSKI T G, BRANHAM M S, DAHMUS J B,

et al. Thermodynamic analysis of resources used in manufacturing processes[J]. Environmental Science and Technology, 2009, 43(5): 1584-1590.

[5] NEUGEBAUER R, WABNER M, RENTZSCH H, et al. Structure principles of energy efficient machine tools[J]. CIRP Journal of Manufacturing Science and Technology, 2011, 4(2): 136-147.

[6] LI C, CHEN X, TANG Y, et al. Selection of optimum parameters in multi-pass face milling for maximum energy efficiency and minimum production cost[J]. Journal of Cleaner Production, 2017, 140(3): 1805-1818.

[7] LI C, XIAO Q, TANG Y, et al. A method integrating Taguchi, RSM and MOPSO to CNC machining parameters optimization for energy saving[J]. Journal of Cleaner Production, 2016, 135: 263-275.

[8] DAI M, TANG D, XU Y, et al. Energy-aware integrated process planning and scheduling for job shops[J]. Proceedings of the Institution of Mechanical Engineers, Part B: Journal of Engineering Manufacture, 2014, 229(Suppl.1): 13-26.

[9] LIN W, YU D Y, ZHANG C, et al. A multi-objective teaching-learning-based optimization algorithm to scheduling in turning processes for minimizing makespan and carbon footprint[J]. Journal of Cleaner Production, 2015, 101: 337-347.

[10] LI L, LI C, TANG Y, et al. An integrated approach of process planning and cutting parameter optimization for Energy-aware CNC Machining[J]. Journal of Cleaner Production, 2017, 162: 458-473.

[11] 刘琼, 周迎冬, 张渏. 面向低碳的切削参数与调度集成 优化 [J]. 机械工程学报, 2017, 53(5): 24-33.

LIU Qiong, ZHOU Yingdong, ZHANG Yi. Integrated optimization of cutting parameters and scheduling for reducing carbon emissions[J]. Journal of Mechanical Engineering, 2017, 53(5): 24-33.

[12] ÖZGÜVEN C, ÖZBAKIR L, YAVUZ Y. Mathematical models for job-shop scheduling problems with routing and process plan flexibility[J]. Applied Mathematical Modelling, 2010, 34(6): 1539-1548.

[13] JIN L, TANG Q, ZHANG C, et al. More MILP models for integrated process planning and scheduling $[\mathrm{J}]$. International Journal of Production Research, 2016, 54(14): 1-16.

[14] WEI T, KHOSHNEVIS B. A linearized polynomial mixed integer programming model for the integration of process planning and scheduling[J]. Journal of Intelligent Manufacturing, 2004, 15(5): 593-605.

[15] MANNE A S. On the job-shop scheduling problem[J]. Operations Research, 1960, 8(2): 219-223.

[16] LI X, GAO L, SHAO X, et al. Mathematical modeling and evolutionary algorithm-based approach for integrated process planning and scheduling $[\mathrm{J}]$. Computers and Operations Research, 2010, 37(4): 656-667.

[17] ZHANG Z, TANG R, PENG T, et al. A method for minimizing the energy consumption of machining system: Integration of process planning and scheduling[J]. Journal of Cleaner Production, 2016， 137: 1647-1662.

[18] JIN L, ZHANG C, SHAO X, et al. A multi-objective memetic algorithm for integrated process planning and scheduling[J]. International Journal of Advanced Manufacturing Technology, 2015, 85(5): 1-16.

[19] ZHANG C, JIANG P, ZHANG L, et al. Energy-aware integration of process planning and scheduling of advanced machining workshop $[\mathrm{J}]$. Proceedings of the Institution of Mechanical Engineers, Part B: Journal of Engineering Manufacture, 2015, 231(11): 2040-2055.

[20] JIN Liang, ZHANG Chaoyong, SHAO Xinyu. An effective hybrid honey bee mating optimization algorithm for integrated process planning and scheduling problems $[\mathrm{J}]$. The International Journal of Advanced Manufacturing Technology, 2015, 80(5-8): 1253-1264.

[21] UNLU Y, MASON S J. Evaluation of mixed integer programming formulations for non-preemptive parallel machine scheduling problems[J]. Computers and Industrial Engineering, 2010, 58(4): 785-800.

[22] NADERI B, GOHARI S, YAZDANI M. Hybrid flexible flowshop problems: Models and solution methods[J]. Applied Mathematical Modelling, 2014, 38(24) : 5767-5780.

[23] WANG S, LU X, LI X X, et al. A systematic approach of process planning and scheduling optimization for sustainable machining[J]. Journal of Cleaner Production, 2015, 87(1): 914-929.

[24] HE Y, LIU B, ZHANG X, et al. A modeling method of task-oriented energy consumption for machining manufacturing system[J]. Journal of Cleaner Production, 2012, 23(1): 167-174.

[25] 何彦, 王乐祥, 李育锋, 等. 一种面向机械车间柔性工 艺路线的加工任务节能调度方法 $[\mathrm{J}]$. 机械工程学报, 2016, 52(19): 168-179.

HE Yan, WANG Lexiang, LI Yufeng, et al. A scheduling method for reducing energy consumption of machining job shops considering the flexible process plan[J]. Journal 
of Mechanical Engineering, 2016， 52(19): 168-179.

[26] 刘琼, 梅侦. 面向低碳的工艺规划与车间调度集成优化 [J]. 机械工程学报, 2017, 53(11): 164-174.

LIU Qiong, MEI Zhen. Integrated optimization of process planning and shop scheduling for reducing manufacturing carbon emissions[J]. Journal of Mechanical Engineering, 2017, 53(11): 164-174.

[27] 李聪波, 沈欢, 李玲玲, 等. 面向能耗的多工艺路线柔 性作业车间分批优化调度模型 $[\mathrm{J}]$. 机械工程学报, 2017, 53(5): 12-23.

LI Congbo, SHEN Huan, LI Lingling, et al. A batch splitting flexible job shop scheduling model for energy saving under alternative process plans[J]. Journal of Mechanical Engineering, 2017, 53(5): 12-23.

[28] CHE A, WU X, PENG J, et al. Energy-efficient bi-objective single-machine scheduling with power-down mechanism[J]. Computers and Operations Research, 2017, 85: 172-183.

[29] YILDIRIM M B , MOUZON G. Single-machine sustainable production planning to minimize total energy consumption and total completion time using a multiple objective genetic algorithm[J]. IEEE Transactions on Engineering Management, 2012， 59(4): 585-597.

[30] LIU C, YANG J, LIAN J, et al. Sustainable performance oriented operational decision-making of single machine systems with deterministic product arrival time[J]. Journal of Cleaner Production, 2014, 85: 318-330.

[31] LIANG P, YANG H, LIU G, et al. An Ant optimization model for unrelated parallel machine scheduling with energy consumption and total tardiness[J]. Mathematical Problems in Engineering, 2015, 2015: 1-8.
[32] MAY G, STAHL B, TAISCH M, et al. Multi-objective genetic algorithm for energy-efficient job shop scheduling[J]. International Journal of Production Research, 2015, 53(23): 7071-7089.

[33] ZHANG L, TANG Q, WU Z, et al. Mathematical modeling and evolutionary generation of rule sets for energy-efficient flexible job shops[J]. Energy, 2017, 138: 210-227.

[34] WANG J, LI S, LIU J. A multi-granularity model for energy consumption simulation and control of discrete manufacturing system[C] //2012 IEEE 19th International Conference on Industrial Engineering and Engineering Management, October 27-29, 2012, Changsha. Heidelberg: Springer, 2012: 1614-1618.

[35] MENG Leilei, ZHOU Mengchu, ZHANG Chaoyong, et al. A new model for predicting power consumption of machining processes: A turning case[C]// 2016 IEEE International Conference on Automation Science and Engineering (CASE), August 21-25, 2016, TX, Fort Worth. New York: IEEE, 2016: 1289-1294.

[36] WAGNER H M. An integer linear-programming model for machine scheduling[J]. Naval Research Logistics Quarterly, 1959, 6(2): 131-140.

[37] CHAO-HSIENPAN. A study of integer programming formulations for scheduling problems[J]. International Journal of Systems Science, 1997, 28(1): 33-41.

作者简介: 孟否否, 男, 1991 年出生, 博士研究生。主要研究方向为车 间调度、刀具磨损、绿色制造。

E-mail: mengleilei@hust.edu.cn 张超勇(通信作者), 男, 1972 年出生, 博士, 副教授, 硕士研究生导师。 主要研究方向为车间调度、网络化制造和绿色制造。

E-mail: zcyhust@mail.hust.edu.cn 